\title{
Research Article \\ Performance Analysis of Reuse Distance in Cooperative Broadcasting
}

\author{
Jimmi Grönkvist, Anders Hansson, and Arwid Komulainen
}

FOI, Swedish Defence Research Agency, Box 1165, 58111 Linköping, Sweden

Correspondence should be addressed to Anders Hansson; anders.hansson@foi.se

Received 29 January 2016; Revised 29 August 2016; Accepted 16 October 2016

Academic Editor: Gianluigi Ferrari

Copyright ( 2016 Jimmi Grönkvist et al. This is an open access article distributed under the Creative Commons Attribution License, which permits unrestricted use, distribution, and reproduction in any medium, provided the original work is properly cited.

Cooperative broadcasting is a promising technique for robust broadcast with low overhead and delay in mobile ad hoc networks. The technique is attractive for mission-oriented mobile communication, where a majority of the traffic is of broadcast nature. In cooperative broadcasting, all nodes simultaneously retransmit packets. The receiver utilizes cooperative diversity in the simultaneously received signals. The retransmissions continue until all nodes are reached. After the packet has traveled a specific number of hops out from the source, denoted as reuse distance, the source node transmits a new broadcast packet in the time slot used for the previous broadcast packet. If the reuse distance is too small, interference causes packet loss in intermediate nodes. In the literature, a reuse distance of three is common. With an analysis based on a realistic interference model and real terrain data, we show that a reuse distance of at least four is necessary to avoid packet loss in sparsely connected networks, especially for high spectral efficiencies. For frequency hopping, widely used in military systems, we propose a novel method. This method almost eliminates interference for a reuse distance of three, increasing the throughput by $33 \%$ compared to systems with a reuse distance of four.

\section{Introduction}

The intended application in this paper is mobile collaborative missions without preinstalled infrastructure, where the participants are mobile and are working together on a task to achieve shared goals. Examples of such mission-oriented networks are mobile ad hoc networks for military and safety applications. In such networks, efficient broadcast is more important than efficient unicast. In broadcasting, a transmitted packet is expected to reach all nodes in the network through one or more transmissions.

As a large proportion of the traffic in these types of networks is of broadcast nature, it is important that the traffic is sent in a robust and efficient manner to maximize the use of the network. Cooperative broadcast is a promising technique for addressing many challenges in ad hoc networks, such as robust communication under high mobility and transmission of time-critical traffic. In cooperative broadcasting, all nodes simultaneously retransmit each received packet. These transmissions do not result in collisions in the traditional sense.
On the contrary, the receiver utilizes the cooperative diversity in the simultaneously received signals. The retransmissions continue until all nodes in the network are reached. Unicast traffic can be transmitted in the same way, which may be less efficient than traditional ad hoc networks, but this is a minor drawback in networks with mostly broadcast traffic.

An initial approach to cooperative broadcasting was described in [1]. The idea is that all nodes retransmit every received signal. In that way, a source node triggers a large number of signals that form what is called an opportunistic large array (OLA). Subsequently, the approach in [1] has been further studied and developed in several papers, for example, [2-6]. The name concurrent cooperative transmission (CCT) is also used, for example, in $[7,8]$.

In [9], a new ad hoc network architecture including cooperative transmission is proposed. In particular, cooperative broadcasting exploits what is emphasized in [9] as key parts of the new architecture: the cooperative transport of packets and access to the medium for the entire path. 
In [10], a time slot structure allowing cooperative broadcasting (i.e., transmissions occur in specific time slots and all retransmissions occur at the same time) is introduced. The time slot structure enables autonomous cooperative communication at the physical layer and thus extends the results on cooperative diversity in [11]. In [12] the name Barrage Relay Networks (BRN) is introduced. BRN differs from OLA mainly by having coarse time synchronization and more extensive receiver signal processing, allowing transmissions to be many bits long rather than single symbols. In [13] it is shown that the broadcast capacity of a cooperative broadcast solution is high compared to multipoint relay (MPR) flooding.

When transmitting larger files or in cases when the network gets highly loaded, it becomes necessary to maximize the throughput of the network. In such cases, the reuse distance becomes important, that is, the distance in network hops between nodes using the same time slot to send different packets originating from the same source node. Each additional hop requires an extra retransmission, with a corresponding decrease in throughput. It is therefore essential that the reuse distance is kept as small as possible. Most studies of the cooperative broadcast are based on the protocol interference model [14] (a graph model). In this model the minimum reuse distance is three. However, using the more realistic physical-interference model [14] (signal-to-noiseand-interference ratios) the needed reuse distance is harder to predict analytically due to the time-varying interactions between interfering transmitters.

Multipacket interference due to time slot reuse in OLA is investigated in $[15,16]$. The authors study high-density networks using a so-called continuum model, in which the node density tends to infinity while the transmission power per unit area is kept constant. The papers present both theoretical and numerical results for optimal reuse distance under the given system model, in [15] for disc networks and in [16] for strip-shaped networks.

In this paper we study how the reuse distance affects system performance in terms of robustness and system capacity. The study is based on network simulations using a realistic terrain-based channel model that allows the study of complex interference effects present in a real scenario. The focus of the study is smaller networks, typical of mobile military networks. We study networks ranging from sparsely to fully connected, to see how the network connectivity affects the interference from time slot reuse. Further, we investigate the use of varying spectral efficiency and how it can be cooptimized with the reuse distance to improve the capacity of the network.

The rest of the paper is outlined as follows. In Section 2, the concept of cooperative broadcasting is described in more detail. We present the time slot structure and the interference model that the analysis is based on. The section concludes with an intraflow interference analysis. In Section 3, we propose an extension of the presented cooperative broadcasting protocol to a scenario where frequency hopping radios are used and show how frequency hopping can be used to improve robustness. In Section 4, the setup used for network simulations with cooperative broadcasting is presented, and the results of those simulations are presented in Section 5. The results illustrate how the reuse distance affects important network metrics such as robustness and capacity. Finally, the paper is concluded in Section 6, where we present some key insights into how to choose a proper reuse distance, given certain sets of conditions and requirements.

\section{Cooperative Broadcasting}

This section describes cooperative broadcasting, based on the time slot structure of BRN [12]. The time slot structure can be described in the following way. Packets are transmitted in time slots in a fixed time frame that is repeated continuously. Furthermore, we assume that the set of all time slots in the time frame or possibly a part of this set is partitioned into equally sized subsets of time slots, called cooperative broadcast slots (CB slots), which we define as follows.

Definition 1. Given two integers $I$ and $D$ satisfying that $I D$ is not larger than the number of time slots in the time frame, we say that the sets of time slots $S_{i}$,

$$
\mathbf{S}_{i}=\left\{S_{i 1}, \ldots, S_{i D}\right\}, \quad i=1, \ldots, I,
$$

are CB slots if they are mutually disjoint and if each time slot $S_{i,(j-1)}$ precedes the time slot $S_{i j}$, for all indices $i=1, \ldots, I$ and $j=2, \ldots, D$.

How the time slots are grouped into CB slots is predefined and does not change over time. The $\mathrm{CB}$ slots may be scheduled, that is, associated with source nodes, dynamically to different source nodes over time or preallocated statically. We now describe the broadcast mechanism of one CB slot, $\mathbf{S}_{i}$. A network broadcast of a packet is initiated by the source node that transmits a packet in time slot $S_{i 1}$. All nodes that received the packet in time slot $S_{i,(j-1)}$ and have not already transmitted the packet simultaneously retransmit an identical packet in time slot $S_{i j}$. The retransmissions are repeated for every time slot in $\mathbf{S}_{i}$ until $j=D$. Note that nodes receiving a packet in $S_{i D}$ retransmit the packet in $S_{k 1}$, where $S_{k}$ is the next $\mathrm{CB}$ slot associated with the same source node. In the same time slot, the source node transmits the next packet. Note that the size $D$ of a CB slot is equal to the reuse distance, that is, the distance in network hops between nodes using the same time slot to send different packets originating from the same source node.

In general, a cooperative broadcasting scheme neither has nor needs any knowledge about the topology of the network. Thus the reuse distance $D$ is fixed during operation and does not adapt to network topology changes, even if it would be an advantage. For example, if $D=3$ and the network at some moment is a two-hop network; the last time slot in each CB slot remains but is not used.

The transceivers may not be capable of receiving a packet in a time slot and directly retransmitting it in the next time slot due to hardware limitations. In such a case, a CB slot should consist of nonconsecutive time slots, as is shown in the example in Figure 1 with parameter setting $I=3$ and $D=3$. For simplicity, we assume that the time slots are of 


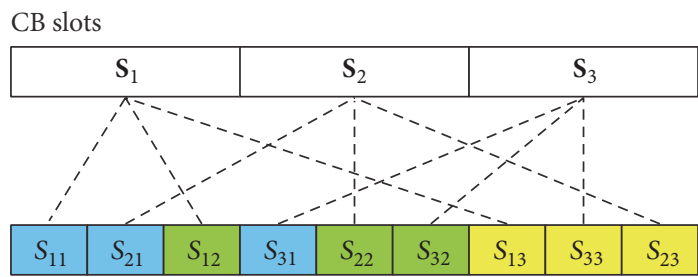

Time slots

FIGURE 1: Illustration of a CB slot partitioning of a frame with nine time slots.

equal lengths and that the number of time slots in the time frame is $I D$.

See Figure 2 for a broadcast example with a CB slot $\mathbf{S}_{1}$ of size three $(D=3)$ containing time slots $S_{11}, S_{12}$, and $S_{13}$. A part of a network is shown in Figure 2(a). A line in the figure represents that direct communication is possible. In Figure 2(b), the source node initiates a broadcast by transmitting a packet in time slot $S_{11}$. Nodes that transmit in time slot $S_{11}$ are blue in Figure 2. In time slot $S_{12}$, the neighbors of the source node, in green, simultaneously retransmit the packet; see Figure 2(c). All nodes that received the packet that was transmitted in time slot $S_{12}$ simultaneously retransmit the same packet in the time slot $S_{13}$, yellow nodes in Figure 2(d). Note that nodes that receive a transmission of a packet they have already transmitted do not transmit it a second time. In each time frame that follows, the source node transmits a new broadcast packet while the previous packets are simultaneously being retransmitted further out in the network; see Figure 2(e).

With the help of one CB slot, a packet is transmitted $D$ hops out from the source node. The following CB slot associated with the same source node distributes the packet $D$ hops further away from the source node, and so on, until all nodes are reached. In the general case, depending on the scheduling, zero, one (as in Figure 2) or more CB slots can be associated with a node within each frame. Each CB slot, however, has at most one node associated with it. Note also that cooperative broadcast is very robust in relation to topology changes because all possible links are utilized for a broadcast.

2.1. Interference Model. Here we describe the physical-interference model, used to determine when nodes can receive packets correctly. We assume that a mobile radio network has $N$ half-duplex radio nodes with associated integer identities from 1 to $N$ and represent the set of nodes by the set of its identities $\mathscr{I}=\{1, \ldots, N\}$. The size of the network is the number of nodes, $N$. For simplicity, we assume that each radio node in the network has an isotropic antenna connected to a receiver with noise power $\mathbf{N}_{R}$ and a transmitter with transmission power $\mathbf{P}_{T}$. Let the sequence of all the elapsing time slots in the time slot structure be numbered with increasing integers; we utilize the numbering as a time measure; that is, for an integer $t$, time $t$ refers to the time slot with number $t$.

Denote by $G(i, j, t)$ the path gain between any two distinct nodes $i$ and $j$, at time $t$. Now consider a broadcast flow of packets $\left(p_{-\infty}, \ldots, p_{\infty}\right)$ originating from a node in $\mathscr{I}$. Let $\mathscr{I}(l, t) \in \mathscr{I}$ be the set of all nodes that transmit packet $p_{l}$ at time $t$. For a node $j \in \mathscr{I}$ receiving packet $p_{l}$ at time $t$, we define the signal-to-interference-and-noise ratio (SINR) as

$$
\gamma(j, l, t)=\frac{\mathbf{P}_{T} \sum_{i \in \mathscr{F}(l, t)} G(i, j, t)}{\mathbf{N}_{R}+\mathbf{P}_{T} \sum_{m \neq l} \sum_{k \in \mathscr{F}(m, t)} G(k, j, t)} .
$$

We assume that a node is able to receive a packet successfully without error if the SINR is not less than a reliable communication threshold, $\gamma_{R}$. The threshold varies depending on spectral efficiency, which is further studied in Section 5.2.

Simultaneous retransmissions require a receiver to be able to combine multiple received identical packets. It resembles the receiver requirements for channels with delay spread and can therefore be handled with the same techniques, as mentioned in [17]. However, the actual receiver technology used for cooperative transmissions does not affect the results of this paper.

2.2. Intraflow Interference Analysis. In this section we show that a large reuse distance $D$ does not guarantee interferencefree networks.

Theorem 2. For any reuse distance $D$ less than $N$, it is possible to construct a network topology with intraflow interference.

We analyse the interference in node 2 in the network shown in Figure 3.

Proof. Assume a network as in Figure 3, with shortest path length between node 3 and node $K$ equal to $D-2$. Let the times $t_{1}, \ldots, t_{2 D}$ correspond to the $2 D$ time slots in two consecutive $\mathrm{CB}$ slots associated with node 1 . At time $t_{1}$, node 1 transmits a packet $p_{l}$. The packet is retransmitted by node 2 at time $t_{2}$ and by node 3 at time $t_{3}$. Assume that the SINR levels $\gamma\left(2, l, t_{1}\right)$ are just above the threshold $\gamma_{R}$ and that $\gamma\left(K, l, t_{2}\right)$ is slightly too low for node $K$ to receive packet $p_{l}$ from node 2 .

At time $t_{D+1}$, as illustrated by the arrows in the figure, node $K$ retransmits the packet $p_{l}$, since it is at distance $D$ from the source, and node 1 transmits the next packet $p_{l+1}$ in the flow.

We now calculate the interference in node 2 at time $t_{D+1}$. The constraints on the SINR levels given by (2) in the scenario can be expressed by considering a small positive value $\varepsilon$ that satisfies the following two equations:

$$
\begin{aligned}
\gamma_{R}<\gamma\left(2, l, t_{1}\right) & =\frac{\mathbf{P}_{T} G\left(1,2, t_{1}\right)}{\mathbf{N}_{R}}<\gamma_{R}+\varepsilon, \\
\gamma_{R}-\varepsilon<\gamma\left(K, l, t_{2}\right) & =\frac{\mathbf{P}_{T} G\left(2, K, t_{2}\right)}{\mathbf{N}_{R}}<\gamma_{R} .
\end{aligned}
$$

If we assume that the path gains are reciprocal and do not change between two consecutive CB slots, that is, $G\left(1,2, t_{1}\right)=$ $G\left(1,2, t_{D+1}\right)$ and $G\left(2, K, t_{2}\right)=G\left(K, 2, t_{D+1}\right)$, then it follows 


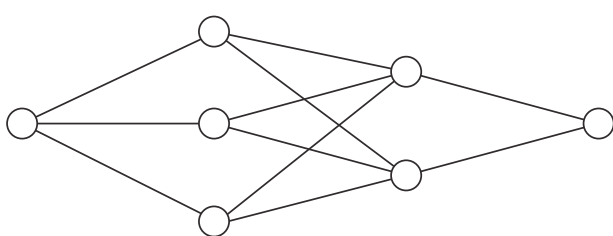

(a)

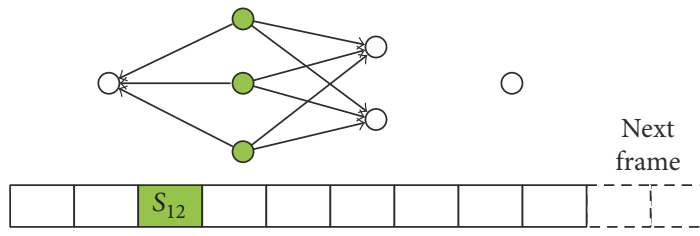

(c)

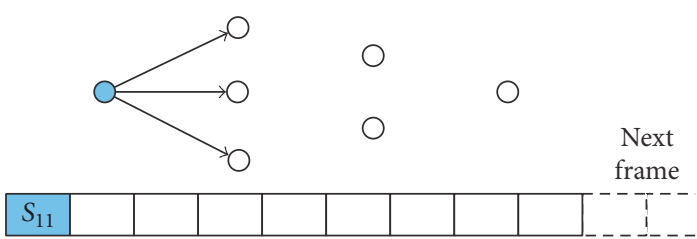

(b)

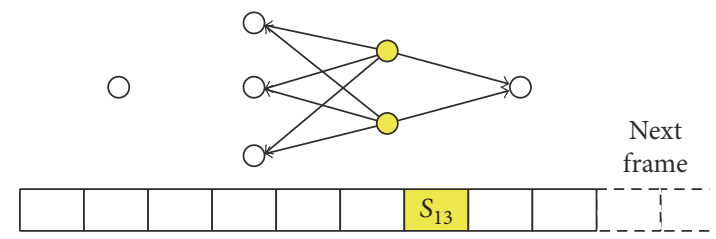

(d)

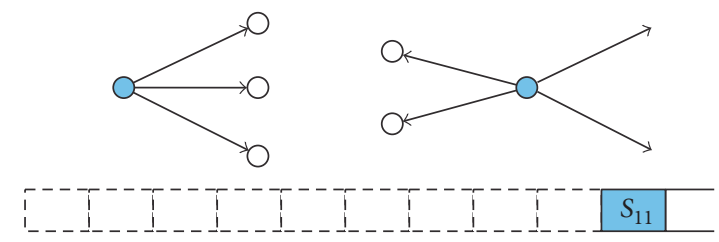

(e)

FIGURE 2: Illustration of cooperative broadcasting.

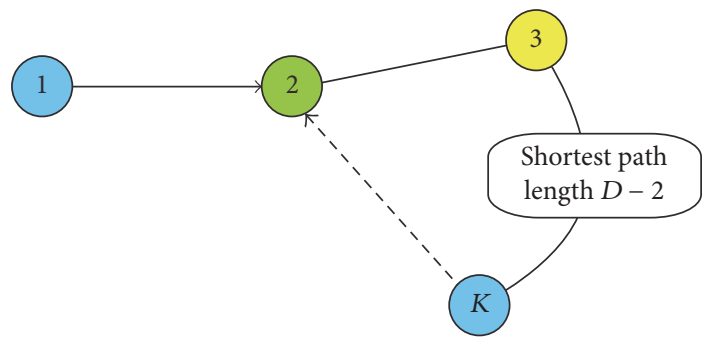

FIGURE 3: Network example for the intraflow interference analysis.

from (3) that the SINR in node 2 falls under the threshold $\gamma_{R}$ in the first time slot of the second CB slot if $\varepsilon$ is too small:

$$
\begin{aligned}
\gamma\left(2, l+1, t_{D+1}\right) & =\frac{\mathbf{P}_{T} G\left(1,2, t_{D+1}\right)}{\mathbf{N}_{R}+\mathbf{P}_{T} G\left(K, 2, t_{D+1}\right)} \\
& <\frac{\mathbf{N}_{R}\left(\gamma_{R}+\varepsilon\right)}{\mathbf{N}_{R}+\mathbf{N}_{R}\left(\gamma_{R}-\varepsilon\right)}=\frac{\gamma_{R}+\varepsilon}{1+\gamma_{R}-\varepsilon} .
\end{aligned}
$$

Since expression (4) is less than $\gamma_{R}$ for a sufficiently small value of $\varepsilon$, we see that the packet $p_{l+1}$ is not received correctly due to interference.

It follows from the theorem that we cannot guarantee interference-free communication, because $D$ is fixed for a given radio system and the network topology is not predictable. Moreover, it should be noted that interference can occur for fairly large values of $\varepsilon$ in the proof. We note that the expression in (4) is less than $\gamma_{R}$ if $\varepsilon$ satisfies the inequality

$$
\varepsilon<\frac{\gamma_{R}^{2}}{1+\gamma_{R}}
$$

and, by combining (5) with (3), we get a condition on the path gains $G\left(1,2, t_{1}\right)$ and $G\left(2, K, t_{2}\right)$ for too high interference in node 2 :

$$
\frac{G\left(1,2, t_{1}\right)}{G\left(2, K, t_{2}\right)}=\frac{\gamma\left(2, l, t_{1}\right)}{\gamma\left(K, l, t_{2}\right)}<2 \gamma_{R}+1 .
$$

In other words, (6) implies that node 2 is unable to receive packets from node 1 due to interference if the path gain $G(2, K, t)$ is too low for direct communication, but larger than a factor $1 /\left(2 \gamma_{R}+1\right)$ of the path gain between node 1 and node 2. As an example, consider the values $\gamma_{R}=2$ and $\gamma_{R}=6$ and a transmission power gain decreasing with the distance raised to the power four. In this case node $K$ still interferes, even when it is positioned up to $50 \%\left(\gamma_{R}=2\right)$ or up to $90 \%\left(\gamma_{R}=6\right)$ further away from node 2 than node 1 .

So, as long as (6) holds, the consequence is that node 2 does not receive packet $p_{l+1}$ in time slot $t_{D+1}$ and therefore packet $p_{l+1}$ will not be delivered to any node. The overall behavior is that every second packet in the packet flow from node 1 is lost to all other nodes.

It is difficult to theoretically determine how often these types of topology constellations occur in reality. They are less likely for higher values of $D$. In addition, when the source node has more than one neighbor, the message can arrive on other paths. Therefore, we use simulations in the rest of the paper to further evaluate the performance of cooperative broadcast.

\section{Dual Channel Cooperative Broadcast}

Frequency hopping, that is, rapidly switching a carrier among many frequency channels, is a widely used technique in radio 


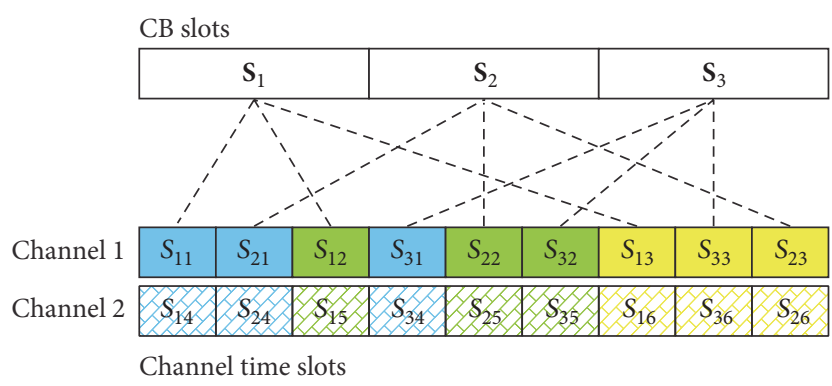

FIGURE 4: Illustration of two-channel cooperative broadcasting. The CB slot $\mathbf{S}_{1}$ is the set of channel time slots $\left\{S_{11}, S_{12}, \ldots, S_{16}\right\}$.

communications. It can be used to make the system more resistant to jamming, to achieve multiple access and to improve the ability to handle fading channels in the receiver. Frequency hopping incurs an extra cost in terms of frequency usage, as the instantaneous bandwidth is only a small part of the total bandwidth used by the system. Within the total frequency band used, multiple communication channels can be achieved by using nonoverlapping frequency hop patterns. When considering a radio system that uses frequency hopping as jamming protection, the interference from nearby transmitting nodes can be reduced by letting some of the nodes use a second additional frequency hopping channel, without increasing the total used frequency band. Using a second frequency-hopping channel does not degrade the jamming protection. In the presence of partial-band jamming, the probability that any of the channels are jammed increases. However, the expected number of jammed receivers does not increase.

Therefore, we suggest a broadcasting method for two channels, both with the same slot structure and with some minor changes compared with the general principles of cooperative broadcasting. Each time slot is mirrored on the second channel so that each two-channel CB slot consists of 6 channel time slots: 3 on the first channel and 3 on the second channel; see Figure 4 and Definition 3.

Definition 3. Assume that we have two channels, channel 1 and channel 2, with $I$ identical CB slots $S_{1}^{\prime}, \ldots, S_{I}^{\prime}$ on each channel: $\mathbf{S}_{i}^{\prime}=\left\{S_{i 1}, S_{i 2}, S_{i 3}\right\}, i=1, \ldots, I$. On channel 2, for $i=1, \ldots, I$, renumber the channel time slots $S_{i 1}, S_{i 2}, S_{i 3}$ to be $S_{i 4}, S_{i 5}, S_{i 6}$, respectively. We define the two-channel $C B$ slots $S_{1}, \ldots, S_{I}$ as the following sets of channel time slots from both channel 1 and channel 2:

$$
\mathbf{S}_{i}=\left\{S_{i 1}, S_{i 2}, S_{i 3}, S_{i 4}, S_{i 5}, S_{i 6}\right\}, \quad i=1, \ldots, I .
$$

A broadcast in a two-channel CB slot $\mathbf{S}_{i}$ starts when the source transmits a packet in channel time $\operatorname{slot} S_{i 1}$ on channel 1. The one-hop neighbors retransmit the packet in channel time slot, $S_{i 2}$, on channel 1 and the two-hop neighbors in channel time slot, $S_{i 3}$, on channel 1. So far nothing is changed compared with single-channel cooperative broadcasting. However, when the three-hop neighbors retransmit the packet, they use the channel time slot $S_{i 4}$ on channel 2, instead of the channel time slot $S_{i 1}$ on channel 1. In this way there is less

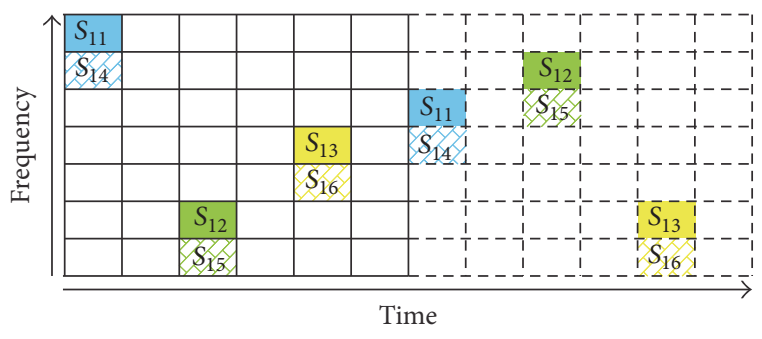

FIGURE 5: Illustration of one CB slot in two channels realized by frequency hopping (in two consecutive frames).

interference with the signal from source transmission on the channel time slot $S_{i 1}$. Four-hop and five-hop neighbors use channel time slot $S_{i 5}$ and channel time slot $S_{i 6}$ on the second channel, which means that the first nodes that reuse the timefrequency slot of the source node are at a six-hop distance.

Figure 5 shows an example of two consecutive time frames (the second time frame is dashed) with one CB slot in two channels realized by frequency hopping patterns. In practice, due to nonperfect frequency filters, traditional implementations often avoid simultaneous transmissions on neighboring frequency channels in a radio system, so that the interference in the receiving nodes is reduced. In our case, however, because the transmitters always are separated by a distance of three network hops, this cochannel interference is sufficiently low to admit simultaneous transmissions on these "free" neighboring frequencies. This is illustrated in the example in the figure, where the time-frequency slot pairs $\left(S_{i 1}, S_{i 4}\right),\left(S_{i 2}, S_{i 5}\right)$, and $\left(S_{i 3}, S_{i 6}\right)$ are scheduled on neighboring frequency channels.

Radio systems that are limited to receiving on one channel at a time can apply the following strategy for finding on which channel to receive the broadcast packets. The initial packet in a broadcast flow is duplicated by the source in two consecutive CB slots. The other nodes alternate between receiving on channel 1 during one CB slot $\left(\left\{S_{i 1}, S_{i 2}, S_{i 3}\right\}\right)$ and on channel 2 in the following CB slot $\left(\left\{S_{i 4}, S_{i 5}, S_{i 6}\right\}\right)$. The broadcast packet is retransmitted in both $\mathrm{CB}$ slots but only one version of the packet is delivered to higher layers. In this way, all nodes pick up one of the duplicated packets. For the following packets in the broadcast flow, the nodes listen to the most likely frequency, based on the channel time slot in which it received the previous packet in the broadcast flow. It is most likely that the next packet in the broadcast flow will be transmitted from the same distance or one hop more or less relative to the previous packet. If, for example, a node receives a packet in time slot $S_{i 3}$ and $\mathbf{S}_{k}$ is the next CB slot associated with the same source node, it listens for the next packet in channel time slots $S_{k 2}, S_{k 3}$, and $S_{k 4}$ as small or no change in distance to the source is more likely than a larger change in distance.

If no packet is received in a succession of CB slots associated with the same source, the node falls back to alternating between the two channels every other CB slot. 


\section{Simulation Setup}

The effects of reuse distance on network performance are examined using network simulations. In this section we present the scenario used and the network metrics considered.

4.1. Scenario. The simulator used for the evaluations is an in-house, packet-based radio network simulator written in $\mathrm{C}++$. The simulations are performed for static networks of varying size and connectivity (measured as the average number of neighbors in the network). Three networks sizes are considered: 30,60 , and 120 nodes, where the main part of the results is for 60 node networks and the other two network sizes are used for complementary results.

The networks are generated by placing nodes randomly in a square with side length $d$. The node density is kept constant at one node per square kilometer for all network realizations, whereas the size of the area is adjusted according to the number of nodes. The path gains between the nodes, $G(i, j, t)$ in (2), are modeled using the propagation library Det-Vag90 ${ }^{\circledR}$, which utilizes a uniform geometrical theory of diffraction (UTD) model by Holm [18]. The path-gain calculations are performed for a terrain-profile contained in a digital database with a resolution of 50 meters. The terrain used is flat rural terrain and the frequency is assumed to be $300 \mathrm{MHz}$. The use of a realistic channel model allows us to investigate the interference effects that cannot be predicted by a pure distance-dependent path-loss model.

Only networks which are connected, in the sense that there exists a path between all pairs of nodes, are considered. To determine connectivity, the channel capacity for a channel perturbed by additive white Gaussian noise [19] is used

$$
\rho=\log _{2}\left(1+\gamma_{R}\right)
$$

where $\rho$ is the spectral efficiency. Two nodes are said to be neighbors if the SNR in the receiver is greater than or equal to $\gamma_{R}$. For a nominal spectral efficiency $\rho_{0}$ of $1 \mathrm{bit} / \mathrm{s} / \mathrm{Hz}$, two nodes are neighbors if the $\mathrm{SNR}$ in the receiver is greater than or equal to $0 \mathrm{~dB}$. For increasing $\rho$ a higher SNR is required for two nodes to be neighbors. Analogously, a packet is determined correctly received if the SINR is greater than or equal to $\gamma_{R}$.

To vary the connectivity of the networks, the transmitting power is adjusted to increase or decrease the average number of neighbors. By only using connected, static networks all packet losses should occur due to interferences from other noncooperating packets, which is what we are mainly interested in.

A static TDMA protocol consisting of CB slots is used on the MAC-layer and each node is given one CB slot per frame, as defined in Definition 1. The proportion of the bandwidth available for transmitting new packets is therefore inversely proportional to the $\mathrm{CB}$ slot size.

The traffic pattern considered is broadcast traffic. Each node generates new packets at a rate equal to the number of frames per second to precisely saturate the channel, where the size of the packets is adjusted to precisely fit one packet in a CB slot.
In the simulations we vary the reuse distance from 3 to 6 hops but we do not explicitly simulate the dual channel solution. It should, however, be noted that the interference sensitivity using the dual channel solution is equal to using 6 slots on a single channel. The capacity, as investigated in Section 5.4, for the dual channel solution is the same as using 3 slots, under the assumption that frequency hopping is already used; see Section 3.

4.2. Performance Metrics. As the traffic transmitted in the simulations is broadcast traffic, we define the Packet Delivery Ratio (PDR) on a system level as

$$
\operatorname{PDR}=\frac{n_{r x}}{n_{t x}(N-1)},
$$

where $n_{t x}$ is the number of transmitted packets, $n_{r x}$ is the number of received packets, and $N$ is the number of nodes. As we are interested in the robustness of the systems and their sensitivity to interference from noncooperating packets, we primarily use PDR for performance evaluation.

The second performance measure considered is system capacity, which we define as

$$
C=\frac{\rho}{D}
$$

This is an upper bound on the throughput of the system and is reached with the selected traffic model. The system capacity measure is used to investigate how to balance reuse distance and spectral efficiency to maximize the total network capacity.

\section{Results}

In this section we evaluate how a number of important network metrics are affected by the reuse distance. To begin with, we illustrate how certain network topology properties affect the amount of interference generated from using a short reuse distance and identify the regime of networks that needs to be studied. For the networks that are considered we compare the effects of interference when varying the spectral efficiency. Further, the interference effects for different network sizes are compared. Finally we indicate how the reuse distance and spectral efficiency can be cooptimized in order to maximize the total throughput.

5.1. Network Diameter. Subsequent packets from a source interfere with each other if too short reuse distance is used, as detailed in Section 2. The amount of intraflow interference generated depends in part on the topology of the network; the longer the paths between the nodes are, the more the interference is generated. Intraflow interference can occur as long as the reuse distance is less than or equal to the network diameter, which we define as the longest path of the shortest paths between any two nodes of the network. Intraflow interference in the case where the reuse distance and network diameter are equal comes from the fact that the protocol presented here uses no topology information; all nodes will therefore always transmit each received packet once, even if 


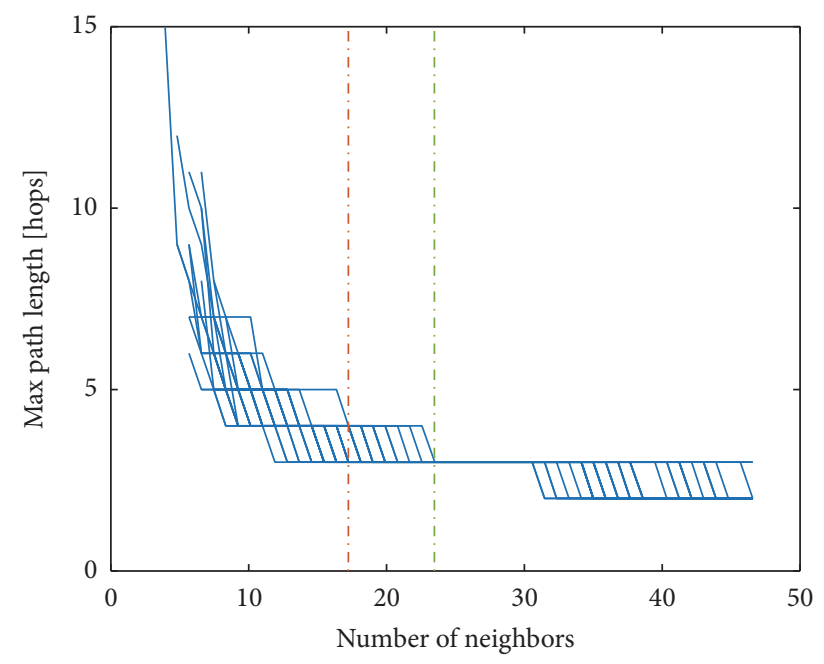

Figure 6: Maximum path length for all 60-node network realizations, in a case with practically no interference.

it has already reached all nodes. When the network diameter increases, we can expect to find more paths that are longer than the reuse distance and therefore increased interference. The network diameter increases as the network becomes more sparsely connected, leading to increased interference problems in sparsely connected networks. In well-connected networks, on the other hand, most nodes will receive the packet within the first or second hop making interference issues only marginal.

In Figures 6 and 7 we show the network diameter as a function of the connectivity of the network, represented by the average number of neighbors, ignoring the interference effects in Figure 6 and using a CB slot size of 3 in Figure 7. In both figures, the red vertical line indicates the region where all networks consist of paths that are 4 hops or less and the green line indicates the region where all paths are 3 hops or less. The network diameter varies a lot in the sparse networks. However, as the red vertical line indicates, at around an average of 17 neighbors we have a maximum of four-hop paths and as the average number of neighbors exceeds 24 (green line) we have a maximum of three-hop paths. As there should be little interference issues in the rightmost region of Figure 6, we focus the rest of the results on the networks where interference from noncooperating packets is possible. As can be seen in Figure 7, the interference caused by previous packets causes the path lengths to slightly increase for sparse networks. We can also see that the limits for three-hop and four-hop networks are pushed further to the right when the interference caused by subsequent packets is accounted for.

5.2. Spectral Efficiency. In this section we study how the spectral efficiency affects the robustness of the system. We consider that a system is robust if the average PDR is above a set threshold, for instance, $99 \%$. The exact percentage needed, of course, depends on the application and the scenario considered.

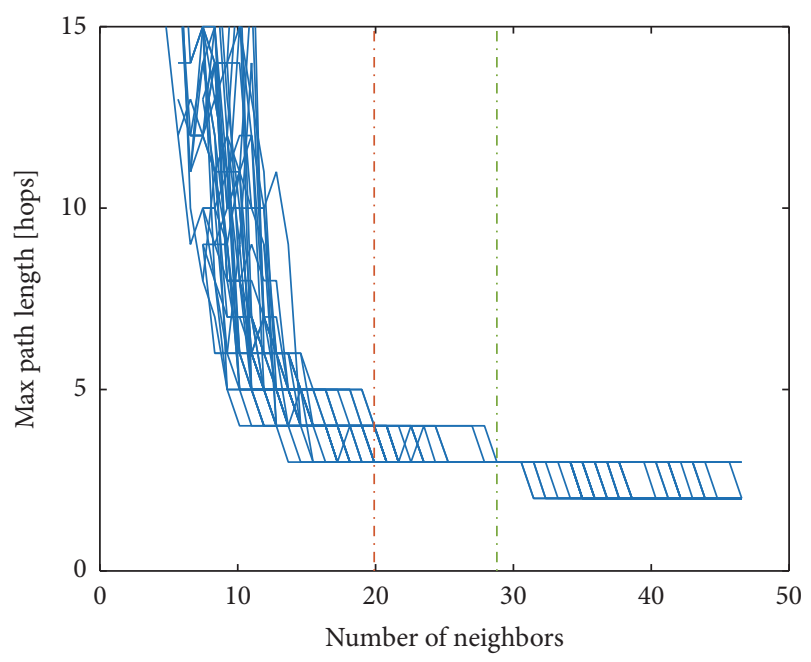

FIGURE 7: Maximum path length for all 60-node network realizations using $\mathrm{CB}$ slots of size 3 .

Communication at high data rates, by the use of higher spectral efficiencies, requires a higher SINR at the receiver, making reception more sensitive to interference. However, as the interference considered here is intraflow interference, increasing the reuse distance should allow the use of higher spectral efficiencies in a robust manner.

The effects of spectral efficiency and reuse distance on system robustness are shown in Figures 8-10. The $y$-axis shows the network averaged PDR, whereas the $x$-axis shows the average number of neighbors at each respective spectral efficiency. Since a higher spectral efficiency requires a higher SNR for two nodes to be neighbors, the transmitting power has been increased proportionally to get the same number of neighbors at varying spectral efficiencies. This is done to isolate the effects of increased interference sensitivity at higher spectral efficiencies and compare the effects of intraflow interference in similar topologies. We see in Figure 8 that already at the nominal spectral efficiency of $1 \mathrm{bit} / \mathrm{s} / \mathrm{Hz}$ a reuse distance of 3 is insufficient when networks become sparse. As expected, increasing the spectral efficiency makes the system less robust, as seen in Figures 9 and 10. To use a spectral efficiency of 4 bits $/ \mathrm{s} / \mathrm{Hz}$, a reuse distance of 6 is required to provide robust communications in sparse networks, due to the increased interference sensitivity.

We note that when the average number of neighbors exceeds 30 , there are practically no interference issues, which is consistent with the results presented in Figure 7 regarding the network diameter.

5.3. Additional Network Sizes. In this section we compare how intraflow interference lowers the robustness of three different network sizes: 30, 60, and 120 nodes. To make the results more comparable between different network sizes, we here show the PDR as a function of the proportion of nodes being neighbors, rather than absolute numbers of neighbors. From Figures 11 and 12 we see that interference from noncooperating packets causes similar problems at all three 


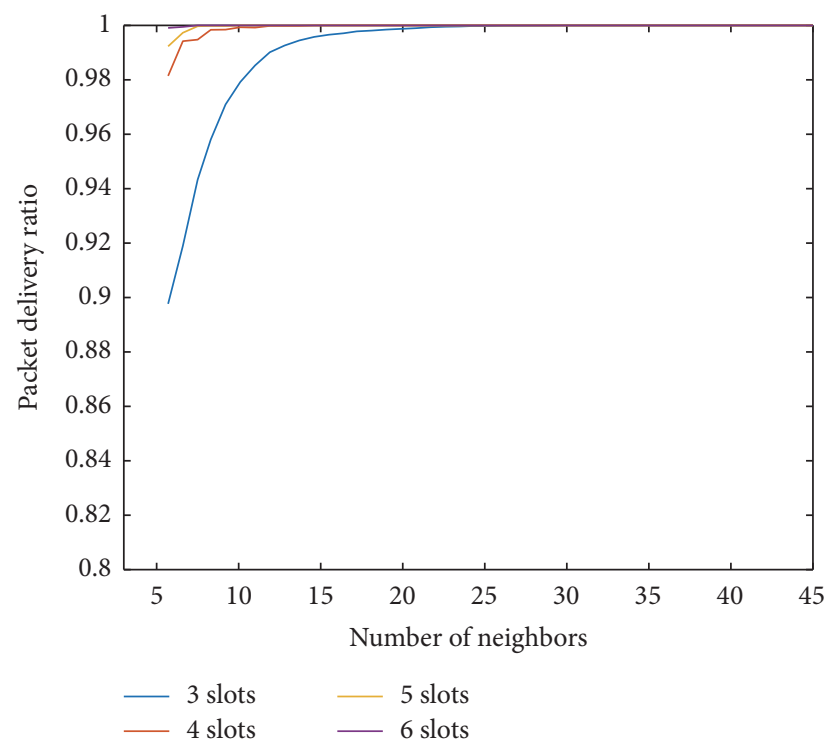

FIGURE 8: Comparison of different reuse distances for nominal data rate $1 \mathrm{bit} / \mathrm{s} / \mathrm{Hz}$.

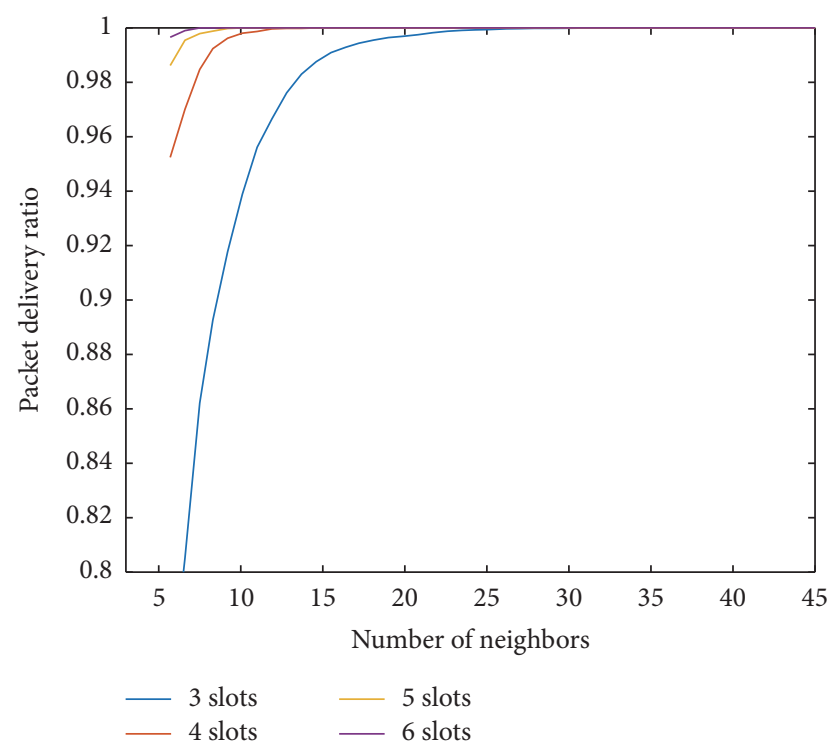

Figure 9: Comparison of different reuse distances for data rate 2 bits/s/Hz.

network sizes, though at slightly different connectivity levels, and we also see that increasing the reuse distance solves the problems in all three cases.

Further, we can note that the interference problems appear to be less in larger networks, at a given neighbor ratio. On one hand the number of possible interferers should be higher in the larger networks causing increased interference problems. On the other hand, more nodes lead to better spatial diversity which seemingly outweighs the increasing number of interferers.

5.4. Capacity. We have now determined that using a low reuse distance causes robustness loss, especially at higher

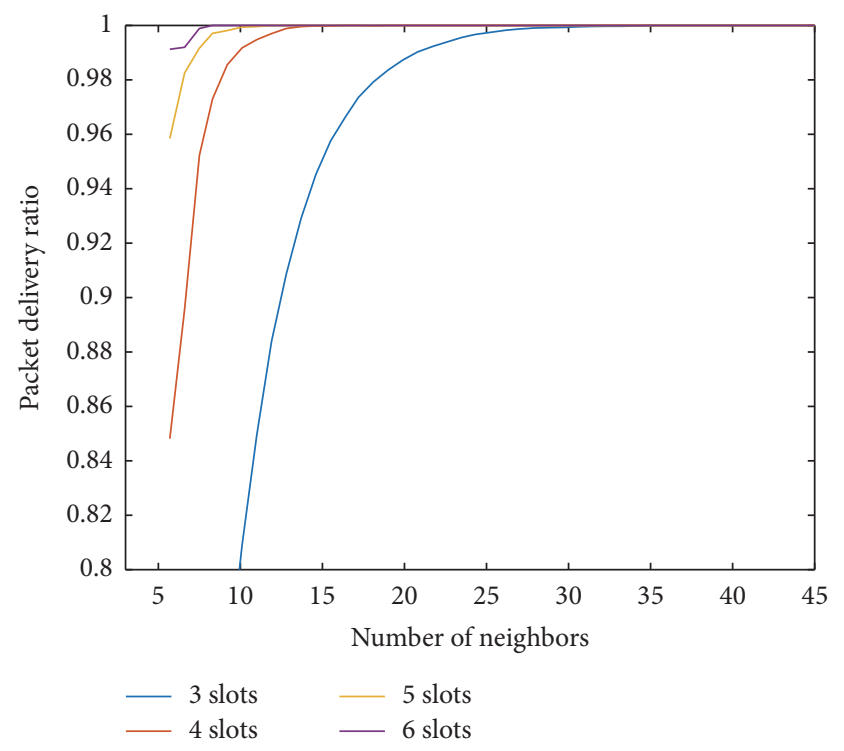

FIGURE 10: Comparison of different reuse distances for highest data rate $4 \mathrm{bits} / \mathrm{s} / \mathrm{Hz}$.

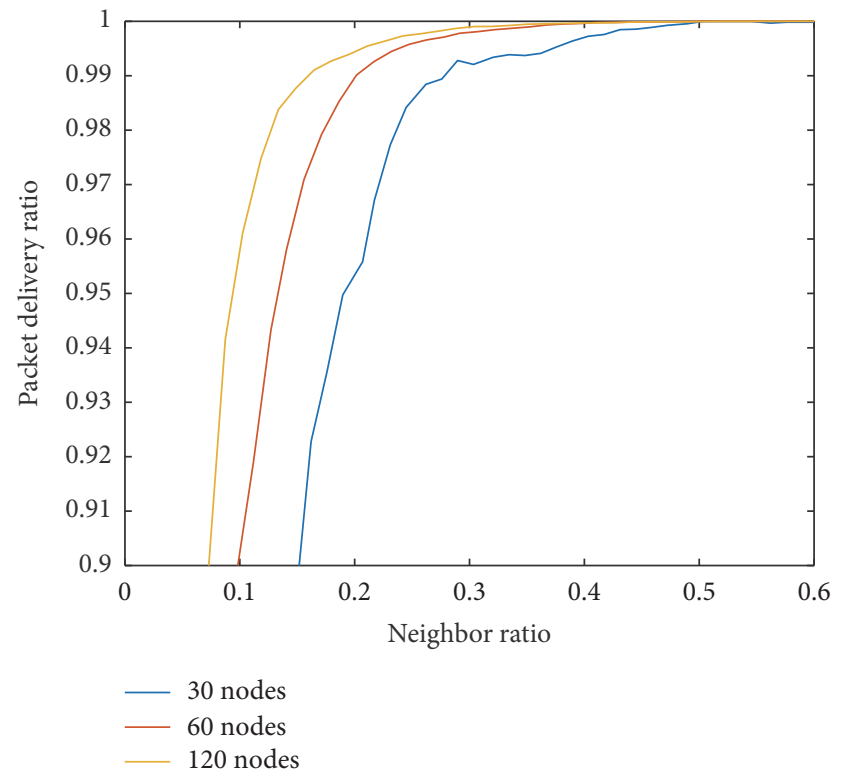

FIgURE 11: Comparison of robustness for different network sizes. Reuse distance 3 hops.

spectral efficiencies. To ensure a robust system, the proposed solution is to increase the reuse distance; generally a reuse distance of at least 4 is needed. However, by increasing the reuse distance the capacity is decreased, if the spectral efficiency used is unchanged. An alternative to increasing the reuse distance is decreasing the spectral efficiency by using a modulation scheme of lower order or increasing coding. A reduction in spectral efficiency lowers the required SINR, resulting in both increasing the amount of interference that can be handled during reception and increasing the connectivity of the network due to longer communication ranges. As shown 


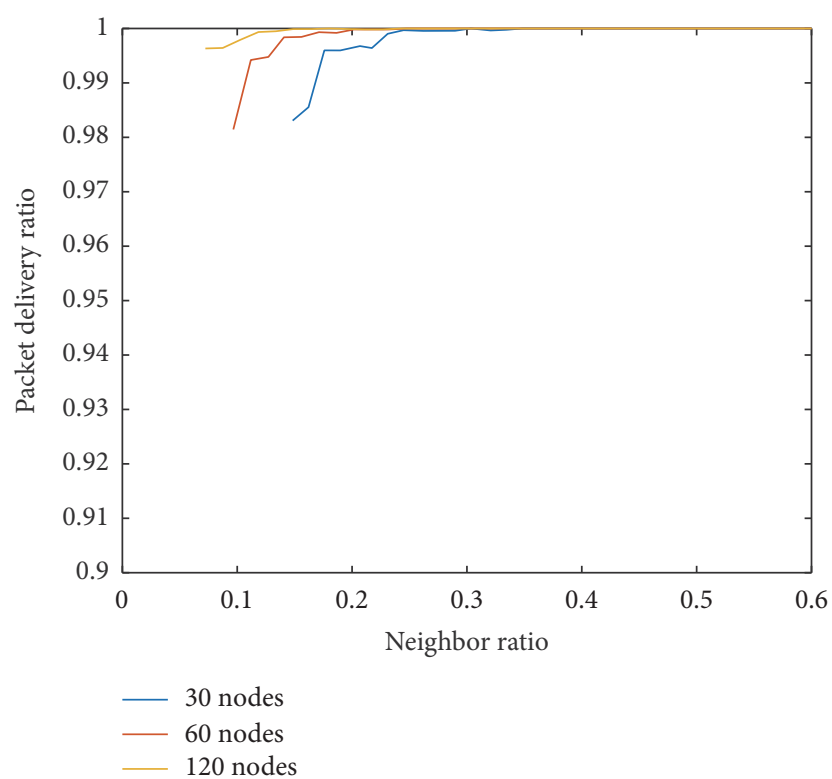

FIgURE 12: Comparison of robustness for different network sizes. Reuse distance 4 hops.

TABLE 1: Minimum and maximum capacity for different reuse distances when spectral efficiency is allowed to vary between 0.5 and 8 bits/s/Hz.

\begin{tabular}{lcc}
\hline$D$ & $C_{\min }$ & $C_{\max }$ \\
\hline 3 & 0.177 & 2.667 \\
4 & 0.125 & 2 \\
5 & 0.1 & 1.6 \\
6 & 0.083 & 1.33 \\
\hline
\end{tabular}

in Section 5.3, as networks become more connected, a cooperative broadcast system becomes more robust.

Based on the above reasoning, we now look at the following problem: given a robustness requirement (e.g., 95\% or 99\% PDR), what combination of reuse distance and spectral efficiency achieves the highest network capacity according to Section 4.2. Figures 13 and 14 present the maximum capacity possible while maintaining a PDR of $95 \%$ and $99 \%$, respectively. The capacity is plotted against the nominal number of neighbors, that is, the number of neighbors when using a spectral efficiency of $1 \mathrm{bit} / \mathrm{s} / \mathrm{Hz}$, since, for these simulations we do not adjust the transmitting power according to the spectral efficiency used, in contrast to the results in Section 5.2. The spectral efficiency is allowed to vary between 0.5 and 8 $\mathrm{bits} / \mathrm{s} / \mathrm{Hz}$. For each combination of reuse distance and spectral efficiency, simulations are run to find the connectivity level at which the robustness demand is fulfilled. These results are combined to get the curves in Figures 13 and 14 to show the highest possible throughput as a function of the network connectivity, for reuse distances ranging from 3 to 6 . In Table 1 the minimum and maximum values for the capacity for each reuse distance are listed for reference.

Comparing the numbers in Table 1 and the curves in Figure 13 we can see that a spectral efficiency of 8 bits $/ \mathrm{s} / \mathrm{Hz}$ is

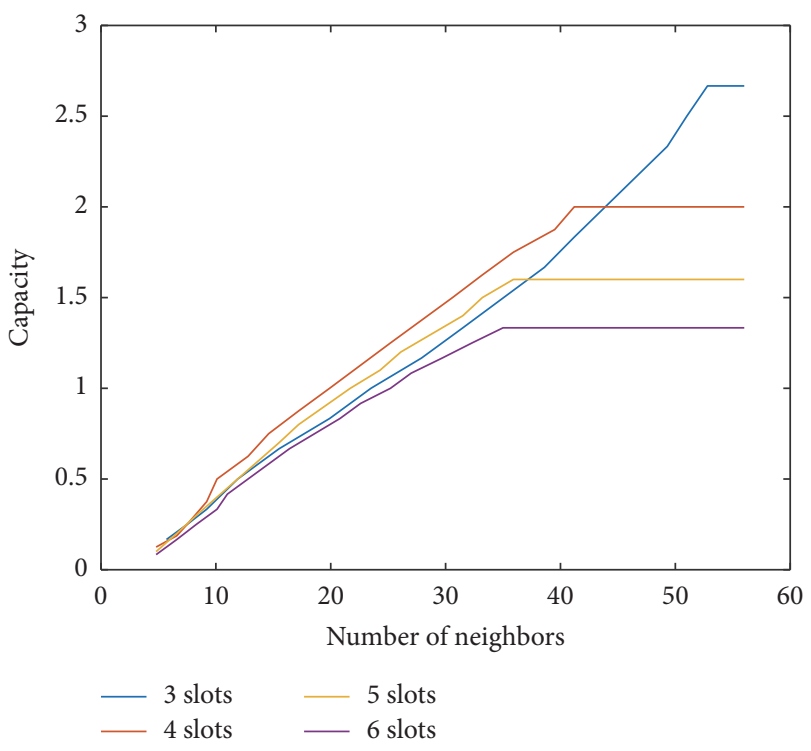

FIgURE 13: Capacity at 95\% robustness.

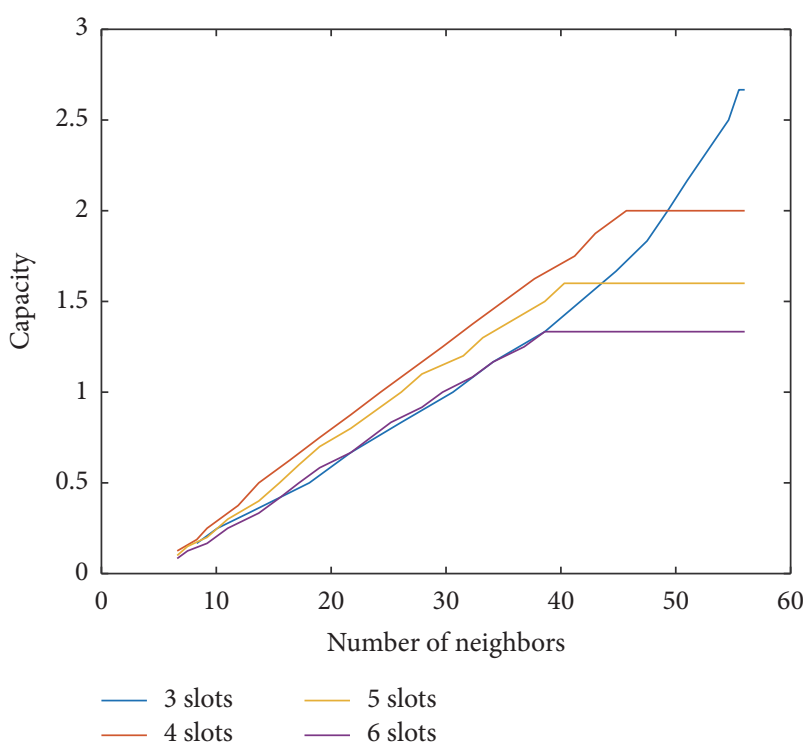

Figure 14: Capacity at $99 \%$ robustness.

achievable at some connectivity level for all reuse distances. Interestingly, irrespective of connectivity level, a reuse distance of 4 appears to be the optimum choice of reuse distance in terms of capacity, except in very well-connected networks. Seemingly, for a given connectivity level, the increase in spectral efficiency that is possible when using a reuse distance of 6 cannot outweigh the loss of capacity incurred by the increased reuse distance.

From Figure 14 we see that the results are similar though somewhat shifted towards the right due to the stricter robustness requirement of $99 \%$ PDR. As was the case for $95 \%$ robustness, a reuse distance of 4 appears to yield the best performance in terms of capacity in all but the most connected networks. 


\section{Conclusions}

Spatial reuse allows geographically separated nodes to schedule concurrent transmissions for a common channel resource. We assess how the performance of cooperative broadcasting is affected by the reuse distance, that is, the distance from the broadcast source node to the nodes that use the same time slot as the source node to retransmit the broadcast traffic, measured in number of hops. The reuse distance should be small, as the network capacity is inversely proportional to the reuse distance. However, if the reuse distance is too low, interference causes packet loss in intermediate nodes.

Our simulations of mobile networks with 30 to 120 nodes, using real terrain data, show that a reuse distance of 3 causes a high level of packet loss in sparsely connected networks. The results indicate that the reuse distance should be at least 4 or even larger for spectral efficiencies greater than $2 \mathrm{bits} / \mathrm{s} / \mathrm{Hz}$.

Comparing different network sizes suggests that a low reuse distance causes similar interference problems irrespective of network size.

If data rates are adapted to the network connectivity a reuse distance of 4 seems optimal for all but the most connected networks, in which nearly all nodes are neighbors. We also suggest a new method for cooperative broadcasting and frequency hopping that eliminate interference for a reuse distance of 3 , thus increasing the throughput by $33 \%$ as compared with cooperative broadcasting with a reuse distance of 4.

\section{Competing Interests}

The authors declare that there is no conflict of interests regarding the publication of this paper.

\section{References}

[1] A. Scaglione and Y.-W. Hong, "Opportunistic large arrays: cooperative transmission in wireless multihop ad hoc networks to reach far distances," IEEE Transactions on Signal Processing, vol. 51, no. 8, pp. 2082-2092, 2003.

[2] H. Jung and M. A. Ingram, "Analysis of spatial pipelining in opportunistic large array broadcasts," in Proceedings of the IEEE Military Communications Conference (MILCOM '11), pp. 991996, Baltimore, Md, USA, November 2011.

[3] Y. J. Chang, H. Jung, and M. A. Ingram, "Demonstration of an OLA-based cooperative routing protocol in an indoor environment," in Proceedings of the 17th Wireless Conference 2011Sustainable Wireless Technologies (European Wireless) (EW'11), pp. 546-553, Vienna, Austria, April 2011.

[4] A. Kailas and M. A. Ingram, "OLA with transmission threshold for strip networks," in Proceedings of the IEEE Military Communications Conference (MILCOM '09), Boston, Mass, USA, October 2009.

[5] A. Kailas and M. A. Ingram, "Investigating multiple alternating cooperative broadcasts to enhance network longevity," in Proceedings of the IEEE International Conference on Communications (ICC '09), pp. 1-5, IEEE, Dresden, Germany, June 2009.

[6] A. Kailas, T. Lakshmi, M. A. Ingram, and L. Thanayankizil, "A simple cooperative transmission protocol for energy-efficient broadcasting over multi-hop wireless. Networks," KICS/IEEE
Journal of Communication and Networks, vol. 10, no. 2, pp. 213220, 2008 .

[7] Y. J. Chang, H. Jung, and M. A. Ingram, "Demonstration of a new degree of freedom in wireless routing: concurrent cooperative transmission," in Proceedings of the 6th Workshop on Hot Topics in Embedded Networked Sensors (HotEmNets '10), pp. 11:1-11:5, Killarney, Ireland, June 2010.

[8] H. Jung, Y. J. Chang, and M. A. Ingram, "Experimental range extension of concurrent cooperative transmission in indoor environments at $2.4 \mathrm{GHz}$," in Proceedings of the 2010 IEEE Military Communications Conference (MILCOM '10), pp. 148153, San Jose, Calif, USA, November 2010.

[9] R. Ramanathan, "Challenges: a radically new architecture for next generation mobile ad hoc networks," in Proceedings of the 11th Annual International Conference on Mobile Computing and Networking (MobiCom '05), pp. 132-139, ACM, 2005.

[10] A. Blair, T. Brown, K. M. Chugg, and M. Johnson, "Tactical mobile mesh network system design," in Proceedings of the Military Communications Conference (MILCOM '07), pp. 1-7, IEEE, Orlando, Fla, USA, October 2007.

[11] D. K. Lee and K. M. Chugg, "A pragmatic approach to cooperative communication," in Proceedings of the IEEE Military Communications Conference (MILCOM '06), pp. 1-7, Washington, DC, USA, October 2006.

[12] A. Blair, T. Brown, K. M. Chugg, T. R. Halford, and M. Johnson, "Barrage relay networks for cooperative transport in tactical manets," in Proceedings of the 2008 IEEE Military Communications Conference (MILCOM '08), pp. 1-7, IEEE, San Diego, Calif, USA, November 2008.

[13] A. Komulainen, J. Grönkvist, and J. Nilsson, "Comparing the capacity of cooperative broadcast to spatial reuse TDMA in ad hoc networks," in Proceedings of the International Conference on Military Communications and Information Systems (ICMCIS '16), pp. 1-7, IEEE, Brussels, Belgium, May 2016.

[14] P. Gupta and P. R. Kumar, "The capacity of wireless networks," IEEE Transactions on Information Theory, vol. 46, no. 2, pp. 388404, 2000 .

[15] H. Jung and M. A. Weitnauer, "Multi-packet interference in opportunistic large array broadcasts over disk networks," IEEE Transactions on Wireless Communications, vol. 12, no. 11, pp. 5631-5645, 2013.

[16] H. Jung and M. A. Weitnauer, "Multi-packet opportunistic large array transmission on strip-shaped cooperative routes or networks," IEEE Transactions on Wireless Communications, vol. 13, no. 1, pp. 144-158, 2014.

[17] T. R. Halford and K. M. Chuggy, "Barrage relay networks," in Proceedings of the Information Theory and Applications Workshop (ITA '10), pp. 153-160, February 2010.

[18] P. D. Holm, "UTD-diffraction coefficients for higher order wedge diffracted fields," IEEE Transactions on Antennas and Propagation, vol. 44, no. 6, pp. 879-888, 1996.

[19] C. E. Shannon, "Communication in the presence of noise," Proceedings of the Institute of Radio Engineers, vol. 37, no. 1, pp. 10$21,1949$. 


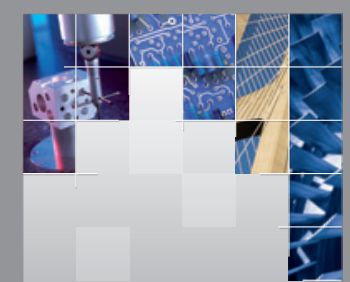

\section{Enfincering}
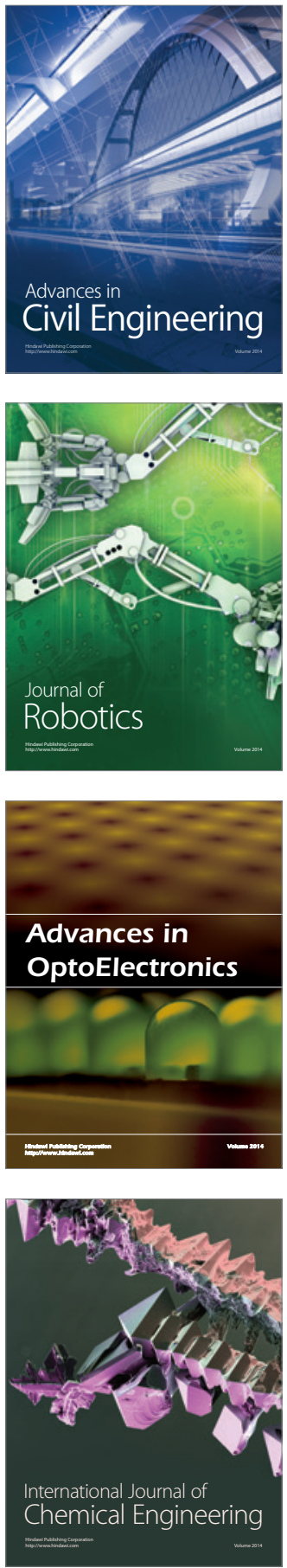

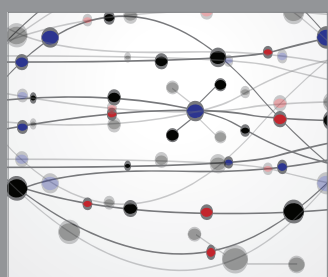

The Scientific World Journal

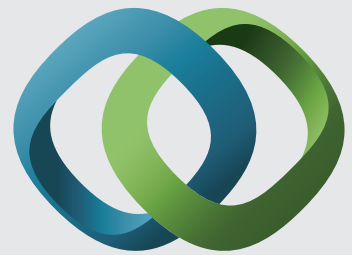

\section{Hindawi}

Submit your manuscripts at

http://www.hindawi.com
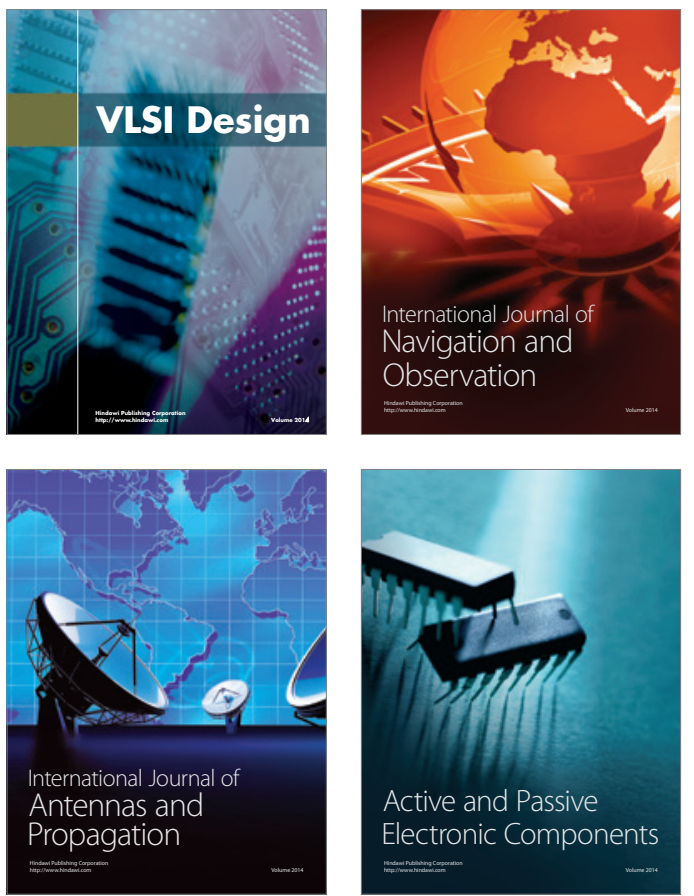
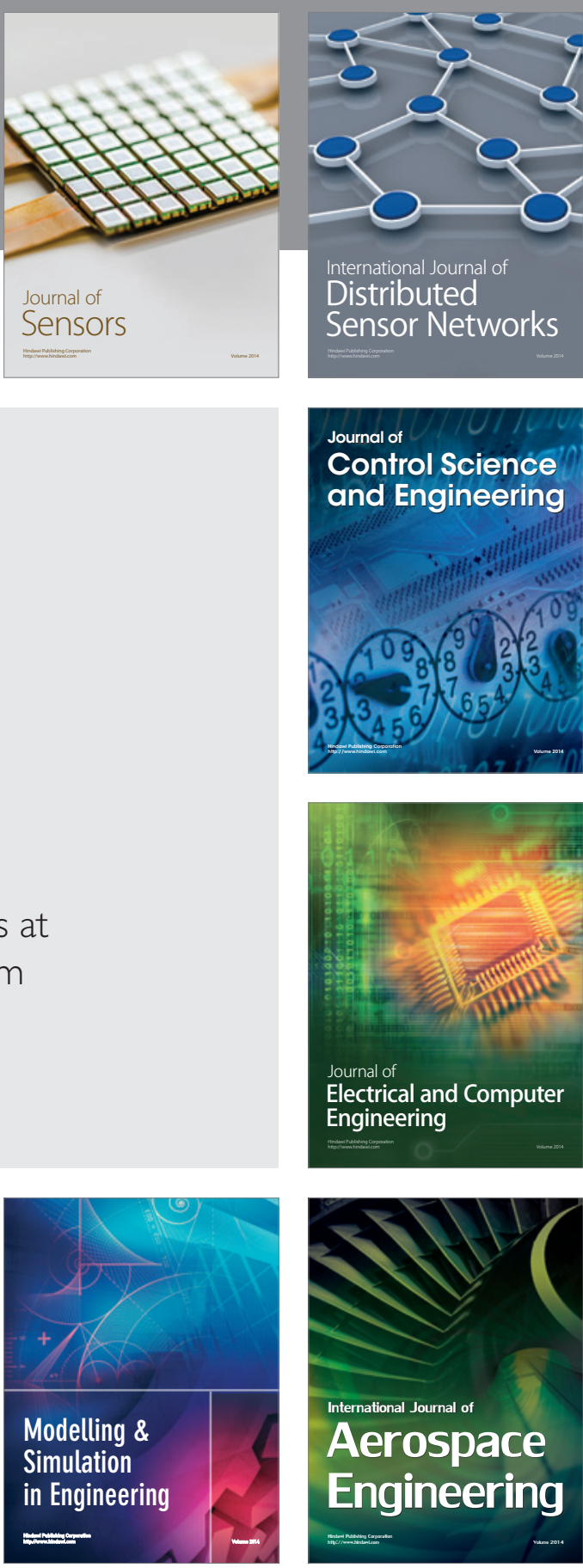

International Journal of

Distributed

Sensor Networks

Journal of

Control Science

and Engineering
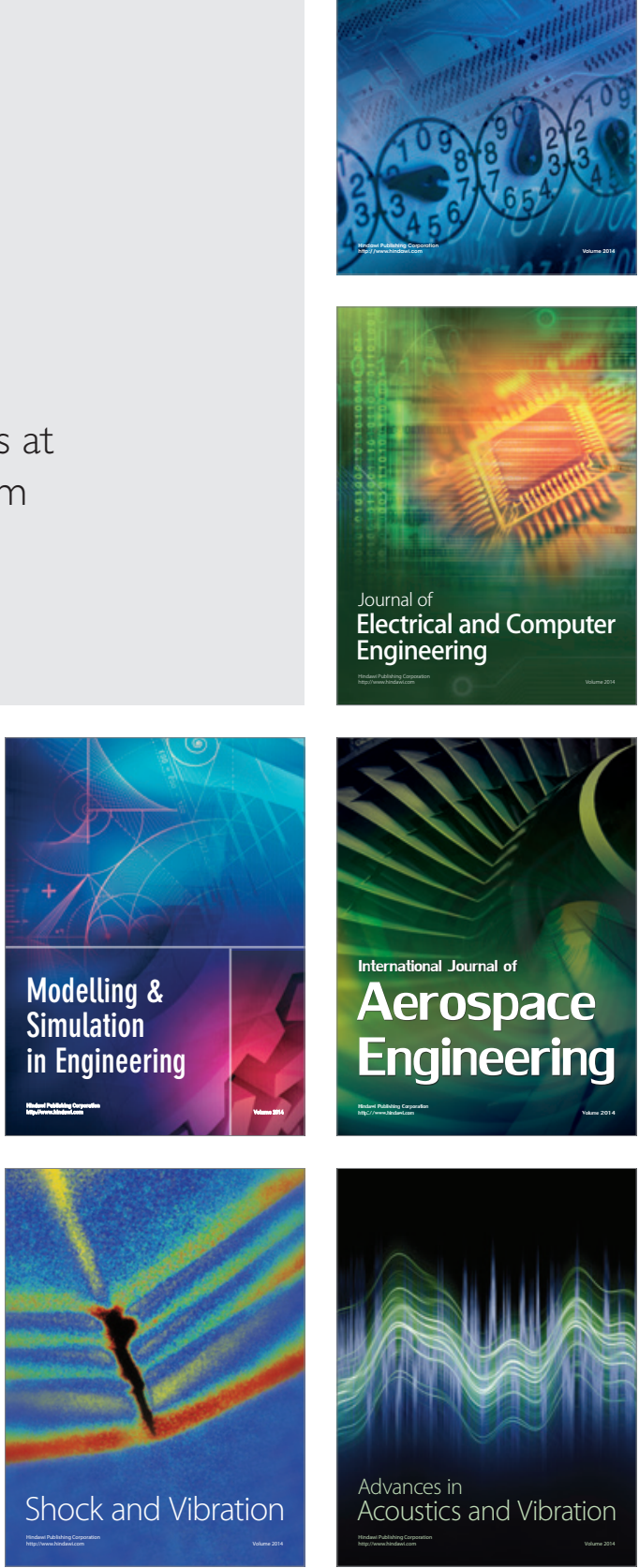